\title{
Modulated phases in multi-stage structural transformations
}

\author{
T. Castán, ${ }^{1}$ A. Planes, ${ }^{1}$ and A. Saxena ${ }^{1,2}$ \\ ${ }^{1}$ Department d'Estructura i Constituents de la Matèria, Universitat de Barcelona, Diagonal 647, 08028 Barcelona, Spain \\ ${ }^{2}$ Theoretical Division, Los Alamos National Laboratory, Los Alamos, New Mexico 87545
}

(Received 19 July 2002; published 28 April 2003)

\begin{abstract}
For multistage displacive structural transitions we present a general framework that accounts for various intermediate modulated phases, elastic constant, phonon, and related thermodynamic anomalies. Based on the presence or absence of modulated phases we classify these transformations in four categories and apply this approach to four different representative materials Ni-Mn-Ga (or $\alpha-\mathrm{U}$ ), NiTi(Fe), Ni-Al, and Cu-Zn-Al. We suggest that the anomalous increase in elastic constants and phonon frequency observed when approaching the martensitic transition from above is a signature of the commensurate modulated phase.

DOI: 10.1103/PhysRevB.67.134113

PACS number(s): 81.30.Kf, 64.70.Kb, 62.20.Dc
\end{abstract}

\section{INTRODUCTION}

A quite varied structural and thermodynamic behavior is observed in a wide class of materials of technological interest such as martensites and shape memory alloys. ${ }^{1} \mathrm{~A}$ displacive structural transition to the low symmetry ("martensite") phase is often preceded by one or more modulated "phases." 2 However, under the vast experimental data, various anomalies and disparate mechanisms, there must be some unifying principles that are common to most of these materials. The present study is an attempt to address this question by identifying and providing a common framework for the different multi-stage transformation mechanisms in displacive martensitic transitions (MT's). This general framework emerges naturally after appropriately assimilating the existing experimental data. The new paradigm being that, concerning structural properties in multistage transformations, very different materials such as magnetic shape memory alloys and actinides (e.g., $\alpha$-U) exhibit similar behavior which can be understood within a single framework or model. The discussion below is restricted to displacive (nonreconstructive) transitions.

The modulated phase is, in general, an incommensurate phase which, eventually, may lock into a commensurate modulation due to the freezing of a specific phonon, usually with an associated wave vector inside the Brillouin zone. To be more precise, the commensurate modulation may or may not be observed but never without a preceding incommensurate modulation. A general scenario for a cubic symmetry parent ("austenite") phase is depicted in Fig. 1. Note that, in general, there may be three transition temperatures. (i) $T_{\mathrm{I}}$ is the temperature at which the incommensurate modulation (IC) first appears; this is (presumably) a second order transition. We note that, in real space, this phase is sometimes referred to as "tweed" in the literature. It has been observed in other materials ${ }^{3}$ such as quartz, high- $T_{c}$ superconducting perovskites, ferroelectrics, etc. (ii) $T_{\mathrm{II}}$ is the temperature at which the commensurate modulation (CM) appears from a (previous) IC phase; this is usually a first order transition. (iii) $T_{M}$ is the first order MT temperature. Depending on the material, either the commensurate phase or both the commensurate and incommensurate phases may not appear. In the latter case there is no modulation or precursor ${ }^{4}$ phenom- ena (either in pure or intermetallic crystals) and a direct transition to the martensitic phase takes place.

Concerning the IC phase ("tweed" in real space) we note that in nonstoichiometric alloys there could be premonitory effects such as thermal expansion anomalies ${ }^{5}$ that are caused by composition fluctuations ${ }^{6}$ and stabilized by long-range elastic forces. ${ }^{7}$

Based on several experimental observations in a variety of materials we first specify the (likely) requirements for a multistage transition.

(a) Existence of low restoring forces along specific directions. In most (cubic) martensitic materials (of interest here) this is accomplished by a temperature softening of the longwavelength limit of the [110][110] transverse acoustic $\left(\mathrm{TA}_{2}\right)$ phonon branch (i.e., $\Sigma_{4}$ branch). This implies that the shear modulus $C^{\prime}=(1 / 2)\left(C_{11}-C_{12}\right)$ is smaller than the other elastic constants. This effect is related to strain.

(b) Phonon softening (dip) observed in [110][1 $1 \overline{1} 0] \mathrm{TA}_{2}$ branch inside the Brillouin zone usually at a particular value of the wavevector $q \neq 0$. This has an effect on shuffle (i.e., intracell distortion) modes.

Hereafter, we shall take these two requirements as necessary (but not sufficient) conditions for applying the point of view adopted in the present study. Besides, they are observed in most martensitic materials ${ }^{8}$ and accepted to be premonitory indications of the low temperature martensitic phase. ${ }^{9}$ Nevertheless, at the MT, neither the elastic constant nor the phonon soften completely. It is now accepted that an incomplete softening is enough to drive the transition. ${ }^{10}$ This is because of an interplay between the strain and the shuffle modes. For onset of the IC, we assume the shuffle mode(s) to be the primary order parameter (OP). Symmetry allowed strain component(s) may then couple to the shuffle as a secondary order parameter, particularly in the CM phase. Depending on the material there may be competition or cooperation between the two effects associated with requirements (a) and (b). In this sense, it is not clear how the interplay between the two partial soft modes operates. In addition, it may be modified by coupling to other physical variables such as magnetism (or inhomogeneities).

This paper is organized as follows. In the next section we provide a new classification of martensitic materials in terms 


\section{$\longleftarrow$ Temperature}

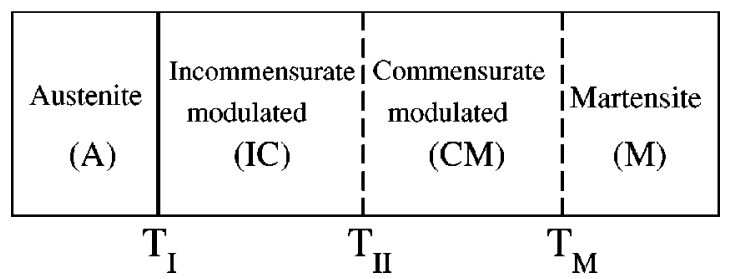

FIG. 1. Paradigm of the multistage displacive structural transitions with modulated precursor phases.

of presence or absence of modulated phases and associated anomalies in phonon dispersion and elastic constants. Section III contains an effective, coupled strain-shuffle, onedimensional model that explains the observed phonon driven anomalies and modulated phases. In Sec. IV we summarize our main findings and propose new experiments that may validate our predictions.

\section{REPRESENTATIVE MATERIALS AND OBSERVED ANOMALIES}

According to the general scenario described above, we can broadly classify various displacive martensitic materials in four categories.

(I) Systems that exhibit both, the IC and CM, intermediate transitions. The Ni-Mn-Ga (Refs. 11-14) and $\alpha$-U (Refs. $15,16)$ materials belong to this category. Both show anomalies in specific heat, ${ }^{16-18}$ magnetic susceptibilities, ${ }^{19}$ resistivity, ${ }^{16}$ and an increase in $C^{\prime}$ with decreasing temperature between $\mathrm{T}_{\mathrm{II}}$ and $\mathrm{T}_{M} \cdot{ }^{15,18,20}$

$\mathrm{Ni}_{2} \mathrm{MnGa}$ is a ferromagnetic Heusler alloy with cubic symmetry and the magnetism (mainly localized on Mn atoms) arises from $d$ electrons. At low temperatures it transforms (for compositions close to stoichiometry) to a tetragonal martensitic phase. ${ }^{21}$ The phonon anomaly is observed at a wave vector $q=\frac{1}{3}[\xi, \xi, 0]$, where $0<\xi<1$.

$\alpha-\mathrm{U}$ is an orthorhombic material and displays two IC phases-incommensurate modulation in one direction or in two directions. The origin of magnetism is (likely to be) the highly directional $f$ electrons in actinides ( $\mathrm{U}$ here). The phonon anomaly is observed at $q=\frac{1}{2}[\xi, 0,0]$. The martensitic phase is presumably monoclinic but this is not yet known although there is a small peak in specific heat recently observed $^{16}$ at $1.2 \mathrm{~K}$.

We notice that for materials in this group, the lock-in transition at $T_{\mathrm{II}}$ occurs without a change in symmetry (at least on an average).

(II) In this second category we include those systems, although they exhibit the two modulated phases, in which the lock-in transition is accompanied by a change in symmetry. As a prototype we take the $\mathrm{NiTi}(\mathrm{Fe})$ material. $^{22-24}$ This shape memory alloy (SMA) has a high temperature cubic structure and exhibits an IC phase. It also locks into a CM phase with trigonal symmetry (i.e., an intermediate $R$ phase) before going into a monoclinic martensite. ${ }^{25}$ Very recent high resolution transmission electron microscopy experiments ${ }^{26}$ in $\mathrm{Ni}-\mathrm{Ti}$ have confirmed the existence of the IC phase. More interesting is the observation that the degree of incommensurability decreases with temperature to finally lock into the (CM) $R$ phase. On the other hand, it has been pointed out that the $R$ phase is a legitimate martensite competing with the monoclinic martensite. ${ }^{27}$ Note that this competing martensitic phases scenario is consistent with our framework. There is a small tendency for $C^{\prime}$ to have an up-turn with decreasing temperature ${ }^{28,29}$ and the phonon anomaly is observed at $q=\frac{1}{3}[\xi, \xi, 0]$. In this case the freezing of the phonon with decreasing temperature (unlike the case I above) is accompanied even by a change in space group symmetry (from cubic to trigonal) related to the underlying phonon displacement amplitude. ${ }^{27}$ Note that stoichiometric $\mathrm{AuCd}$ (Refs. 25,30) also has a $B 2$ to $R$ phase transition similar to $\mathrm{NiTi}(\mathrm{Fe})$.

(III) In this category we include systems in which the CM phase is suppressed $\left(T_{\mathrm{II}}=T_{M}\right)$. This is the case of the $\mathrm{Ni}_{x} \mathrm{Al}_{1-x} \quad(0.45<x<0.63) \quad$ (Refs. 31,32), $\mathrm{Fe}_{1-x} \mathrm{Pd}_{x} \quad(x$ $<0.32$ ) (Refs. 33,34), $\mathrm{Fe}_{3} \mathrm{Pt}$ (ordered) (Ref. 35), and In-Tl (Ref. 36) materials. They exhibit an IC phase (referred to as tweed, in real space) but the CM phase is absent before they undergo a MT. The phonon anomaly in NiAl is observed at $q \simeq \frac{1}{6}[\xi, \xi, 0]$ with a monoclinic martensitic phase.

(IV) In the case of $\mathrm{Cu}$-based shape memory alloys (e.g., $\mathrm{Cu}-\mathrm{Zn}-\mathrm{Al}$ ), no modulated phase is observed. There is a unique phase transition from the high temperature cubic austenite phase directly to the martensitic phase. In phonon dispersion curves there is almost no dip but significantly, the whole $\left(\mathrm{TA}_{2}\right)$ phonon branch has a quite low energy. These materials can be termed as ordinary SMA with entropy driven $^{8,37}$ MT.

We note that materials discussed in the above categories exhibit the same premonitory effects but different structural precursors. ${ }^{4}$ The different transitions are accompanied by anomalies in various physical quantities with different magnitudes.

(i) Softening of a specific phonon branch accompanied by a dip. Figure 2 shows experimental examples for Ni-Mn-Ga (Refs. 13,14) $\alpha$-U (Ref. 38) and Ni-Al (Ref. 32). For the purpose of discussion below, the two cubic alloys Ni-Mn-Ga and $\mathrm{Ni}-\mathrm{Al}$ are shown together and $\alpha-\mathrm{U}$ (orthorhombic) is shown in the inset. Note the appealing similar behavior of phonons in Ni-Mn-Ga and $\alpha$-U. Except for a linear decrease, there is no interesting feature in Ni-Al phonons such as a conspicuous change in slope. The different transitions are denoted by arrows. The increase in energy at low temperature for Ni-Mn-Ga is an indication of the phonon freezing at $T_{\text {II }}$. Presumably the $\alpha-\mathrm{U}$ should also exhibit similar low temperature increase in phonon energy but unfortunately the experimental data is lacking.

(ii) Softening of the relevant elastic constants (i.e., the long-wavelength limit of $\mathrm{TA}_{2}$ phonon branch). An example for $C^{\prime}$ and $C_{44}$ softening as the temperature is decreased to $T_{\text {II }}$ and elastic constant "hardening" upon subsequent cooling $^{18,20}$ to $T_{M}$ for Ni-Mn-Ga is depicted in Fig. 3(a). In contrast, other than a linear decrease there is no structure in the behavior of elastic constants for $\mathrm{Ni}-\mathrm{Al}$, also shown on the same figure for comparison. In Fig. 3(b) we show the temperature variation of three of the relevant orthorhombic elas- 


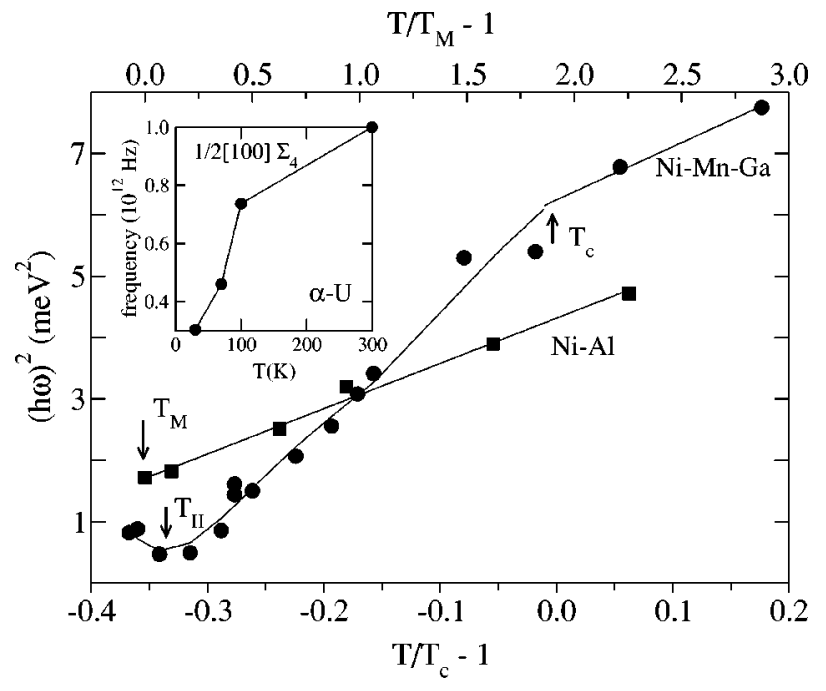

FIG. 2. Energy of the anomalous phonon as a function of reduced temperature for Ni-Al $\left(T_{M}\right)$ and Ni-Mn-Ga $\left(T_{c}\right) . T_{c}$ is the Curie temperature and $T_{I}$ cannot be properly identified. The inset shows the phonon softening in $\alpha$-U. Data have been extracted from references indicated in the text.

tic constants for $\alpha-\mathrm{U}$ (Ref. 39). There is a striking similarity in the $C_{11}$ and $C_{44}$ variation for $\alpha-\mathrm{U}$ and the $C^{\prime}$ and $C_{44}$ variation for Ni-Mn-Ga (Ref. 40). Unfortunately, the $C_{12}$ low temperature data for $\alpha-\mathrm{U}$ is lacking. We also note that a somewhat similar incipient anomaly is observed in $\mathrm{NiTi}(\mathrm{Fe})$ (Ref. 41). In this work, we shall argue that the up-turn (or elastic constant "hardening") with decreasing temperature at $T_{\text {II }}$ is characteristic of systems undergoing multistage modulated structural transformations. This point will be discussed below.

(iii) Anomalies in the specific heat, resistivity, magnetic susceptibility and other thermodynamic variables are also observed. Examples include Ni-Mn-Ga (Ref. 8), NiTi(Fe) (Ref. 23), $\alpha$-U (Ref. 16), and other actinides. ${ }^{42}$

The IC phase is detected by diffuse satellite reflections that appear at incommensurate positions. ${ }^{43}$ Examples include Ni-Mn-Ga (Ref. 11), Ni-Al (Ref. 31), Fe-Pd (Ref. 34), $\mathrm{NiTi}(\mathrm{Fe})($ Refs. 26,44). It is not caused by a phonon instability but it is due to local inhomogeneities ${ }^{6}$ (e.g., compositional fluctuations, crystal defects, residual strain) that (locally) couple to the soft modes. The emerging phase is thermodynamically stabilized by anisotropic, long-range elastic forces. ${ }^{7}$ However, this remains an open question for future investigation. The further lock-in (or freezing) of the phonon at commensurate positions requires an additional degree of softening of the anomalous phonon frequency with decreasing temperature before it reaches the martensitic transition $T_{M}$. It is clear from Fig. 2 that in the case of Ni-Mn$\mathrm{Ga}$, magnetism provides (through a magnetoelastic coupling ${ }^{45}$ ) the enhancement of softening necessary for freezing.

Moreover, it is known that in many martensitic alloys the transition temperature $T_{M}$ is very sensitive to the electron concentration per atom $(e / a)$. This has not been quantified previously, but clearly so. In Fig. 4(a) we compare various transition temperatures for three materials: ${ }^{22} \mathrm{NiTi}$ and
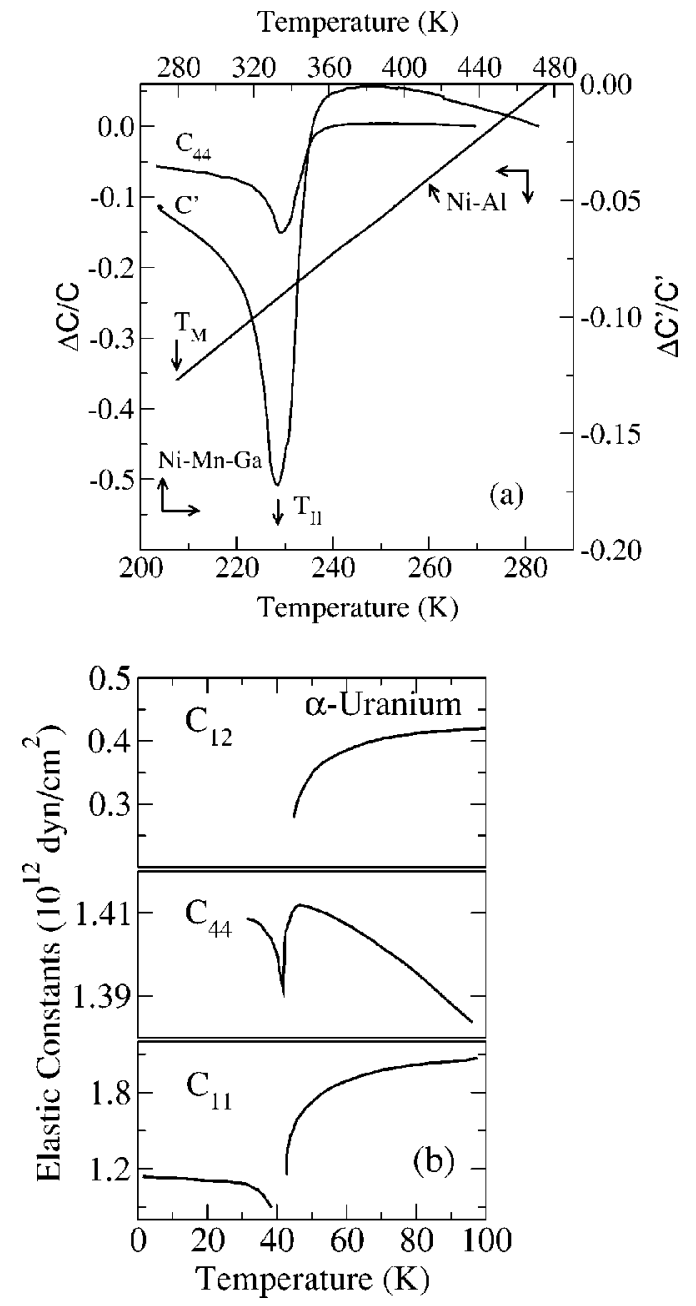

FIG. 3. (a) Elastic constant anomaly in Ni-Mn-Ga compared to that in Ni-Al. (b) Elastic constant anomaly in $\alpha$-U. Data have been extracted from references indicated in the text.

$\mathrm{NiTi}(\mathrm{Fe})$ for two different compositions as a function of $e / a$. (The stable phase for $T_{\mathrm{II}}<T<T_{M}$ is rhombohedral $(R)$ and modulated. ${ }^{27}$ ) This dramatic dependence of lattice stability with the e/a ratio is also observed in Ni-Mn-Ga (Ref. 8) and Fe-Pd (Ref. 46) as well [see Fig. 4(b)]. However, the trend is reversed in Fe-Pd [inset of Fig. 4(b)] because, unlike Ni$\mathrm{Mn}-\mathrm{Ga}$ and Ni-Ti, Fe-Pd is a close-packed (magnetic) structure. Small changes in the relative alloying percentages of the elements may produce significant variations in $T_{M}$ and therefore in the observed behavior of the materials. This is especially relevant in Cu-based alloys and actinides, particularly in $\mathrm{Ga}$ stabilized ${ }^{42} \mathrm{Pu}$. Beyond a certain alloying percentage, the martensitic transformation can be arrested totally (e.g., above $\mathrm{e}^{33,34} 32 \% \mathrm{Pd}$ in Fe-Pd). More precisely, as $T_{M}$ increases, the freezing of the phonon prior to the martensitic transition becomes less likely.

\section{EFFECTIVE 1D MODEL}

From the full three-dimensional crystal symmetry analysis we have a Ginzburg-Landau model in terms of all (six) strain tensor components, ${ }^{47,48}$ all shuffle ${ }^{24}$ order parameters, and 

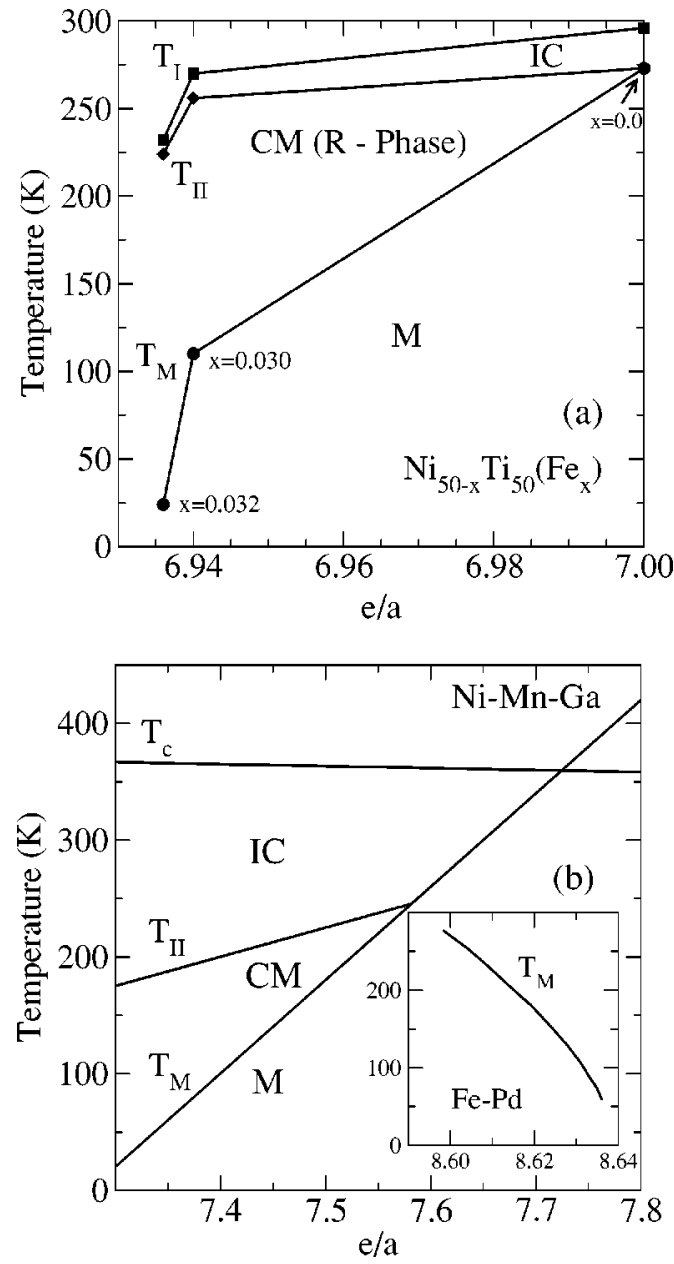

FIG. 4. Various transition temperatures as a function of $e / a$ in (a) Ni-Ti (after Ref. 22), (b) Ni-Mn-Ga (Ref. 8), and in the inset Fe-Pd (Ref. 46).

the magnetization ${ }^{47}$ with the symmetry allowed couplings between strain and shuffle, strain, and magnetization as well as shuffle and magnetization. For specific strain components and the shuffle in a particular direction we can obtain an effective one-dimensional analog of the full 3D model. We emphasize that our goal here is to address the ion-displacive (or phonon-driven ${ }^{49}$ ) anomalies (above $T_{M}$ ) but not the microstructural aspects (for which we need to include the gradient terms ${ }^{50}$ ). Therefore, the effective 1D model is especially appropriate for our purpose.

We focus on the $\vec{q}=[\xi \xi 0]$ projection of the cubic $\rightarrow$ tetragonal distortion. The relevant order parameters are the amplitude of the anomalous phonon $\eta$ and the tetragonal strain $e$ ( $e_{3}$ in standard symmetry-adapted notation). The simplest Landau free-energy expansion is

$$
\begin{aligned}
F(e, \eta)= & \frac{\omega^{2}}{2} \eta^{2}+\frac{\beta}{4} \eta^{4}+\frac{\gamma}{6} \eta^{6}+\frac{C^{\prime}}{2} e^{2}+\frac{A}{3} e^{3}+\frac{B}{4} e^{4} \\
& +\kappa_{e \eta} e \eta^{2},
\end{aligned}
$$

where $\omega$ is the frequency of the anomalous phonon and $C^{\prime}$ the elastic constant defined as $C^{\prime}=\left(C_{11}-C_{12}\right) / 2$. Both soften with decreasing temperature (for the cubic materials considered here). Here $\kappa_{e} \eta$ is a material dependent parameter denoting symmetry allowed coupling between strain and shuffle.

For the analysis of the modulated phases, we write an effective free-energy in terms of the shuffle $\eta$ only, i.e.,

$$
F_{e}(\eta)=\frac{\omega^{2}}{2} \eta^{2}+\frac{\beta_{r}}{4} \eta^{4}+\frac{\gamma}{6} \eta^{6},
$$

with the renormalized coefficient (including fluctuations and anharmonicities ${ }^{10}$ ) being

$$
\beta_{r}=\beta-2\left(\kappa_{e \eta}\right)^{2} / C^{\prime} .
$$

The associated tetragonal distortion is given by ${ }^{51}$

$$
e=-\left(\kappa_{e \eta} / C^{\prime}\right) \eta^{2},
$$

considering only the harmonic terms in strain.

In the IC phase $\beta_{r}>0$ and the last term in the $\eta$ expansion (2) is not relevant (in addition to the shuffle amplitude being very small). One possible origin of this phase is local inhomogeneities that couple to the soft modes ${ }^{6}$ and renormalize the quadratic coefficient so that in some regions the corresponding renormalized phonon frequency $\omega_{r}^{2}$ becomes negative. $T_{\mathrm{I}}$ is the temperature at which anisotropic longrange elastic forces stabilize the IC phase. Then, the intensity of the satellite reflections continuously increases from zero with decreasing temperature and consequently the magnitude of the associated tetragonal strain, which serves as a precursor to martensite.

As the order parameter $\eta$ increases, ${ }^{14,23}$ the sixth-order term in Eq. (2) becomes important. The lock-in of the phonon at commensurate positions requires that $\left(\kappa_{e \eta}\right)^{2} / C^{\prime}$ be large enough so that $\beta_{r}<0$. This is a phonon instability and the transition at $T_{\mathrm{II}}$ is first order.

Subsequent cooling is dominated by the increasing phonon amplitude and the softening of $C^{\prime}$ is not required for the structural instability at $T_{M}$. Actually, materials undergoing the lock-in transition (Ni-Mn-Ga, $\alpha-\mathrm{U}$ ) exhibit a hardening of the relevant elastic constant(s) in the range $T_{M}<T<T_{\text {II }}$ (see Fig. 3). We suggest this unusual behavior (when approaching a structural phase transition) is characteristic of materials for which the phonon instability occurs prior to the structural instability. The upturn at $T_{\text {II }}$ reflects the higher stability of the CM (averaged cubic) phase with respect to the parent cubic phase. The further structural instability results from a symmetry allowed coupling of strain to the increasing value of $\eta$ [that is, from Eq. (4), $\left.e=-\left(\kappa_{e \eta} / C^{\prime}\right) \eta^{2}\right]$.

This analysis may be expanded to include secondary couplings.

(i) Effect of softening in $C_{44}$ : Ren and Otsuka ${ }^{52}$ pointed out that in some materials $C_{44}$ also softens with temperature and consequently there may be a competition between both $C^{\prime}$ and $C_{44}$ modes. In that case,

$$
F(e, \epsilon, \eta)=F(e, \eta)+\frac{C_{44}}{2} \epsilon^{2}+\kappa_{\epsilon \eta} \epsilon \eta^{2},
$$


where $\epsilon$ is the corresponding (symmetry-adapted shear $e_{4}$ ) strain and $\kappa_{\epsilon \eta}$ the strength of the symmetry allowed coupling term. The expression of $F(e, \eta)$ is given by Eq. (1). We may write an effective free-energy in terms of $\eta$ and obtain that the renormalized coefficient is now given by

$$
\beta_{r}=\beta-\frac{2\left(\kappa_{e \eta}\right)^{2}}{C^{\prime}}\left[1+\frac{\left(\kappa_{\epsilon \eta} / \kappa_{e \eta}\right)^{2}}{A}\right],
$$

where $A=C_{44} / C^{\prime}$ is the elastic anisotropy. The coupling between both shear modes is then given by

$$
\epsilon=\left[\frac{\kappa_{\epsilon \eta}}{\kappa_{e \eta}}\right] \frac{e}{A} .
$$

We emphasize that this relationship between the two shear strains is naturally mediated through the elastic constant anisotropy $(A)$.

(ii) Role of magnetism: From the existing data, it appears to renormalize the shuffle coefficients (see Fig. 2). Clearly, $d$ electrons are responsible in $\mathrm{NiTi}(\mathrm{Fe})$ and $\mathrm{Ni}-\mathrm{Mn}-\mathrm{Ga}$ while $f$ electrons are crucial in $\alpha-\mathrm{U}$ and other actinides, especially ${ }^{42,53} \mathrm{Pu}$. The "kink" observed at the Curie point $T_{c}$ is described by writing $\omega_{r}^{2}(m)=\omega^{2}+\kappa_{m} \eta^{m^{2}}$ (with $\kappa_{m \eta}$ $<0$ ), where $m$ denotes magnetization. Other couplings that renormalize the strain coefficients are also very likely. Unfortunately there is no data on the behavior of $C^{\prime}$ around $T_{c}$.

(iii) For $\alpha-\mathrm{U}$ it follows from symmetry analysis that the monoclinic strain is coupled to the frozen phonon amplitude ${ }^{54}$ Thus, the anomaly at $1.2 \mathrm{~K}$ in the specific heat ${ }^{16}$ may be an indication of MT to a monoclinic phase. This may also happen for other actinides, including the apparently strange behavior ${ }^{42,53}$ of $\mathrm{Pu}$.

\section{CONCLUSION}

We have proposed a general framework for understanding multistage martensitic transformations which is consistent with a large amount of experimental data on different materials such as Ni-Al, Ni-Mn-Ga, NiTi(Fe), $\alpha-\mathrm{U}, \mathrm{Fe}-\mathrm{Pd}, \mathrm{Au}-$ $\mathrm{Cd}$, etc. Specifically, presence of an incommensurate, and in some cases an additional commensurate, modulated phase as precursors to the MT reflects itself in the anomalous (softening and further "hardening") of both the elastic constants and phonons. In the case of some magnetic martensites, particularly Ni-Mn-Ga, the phonon softening is enhanced by magnetism. We propose additional experiments that may test the broader validity of our scenario. (i) Measurements of $C^{\prime}$ in Ni-Mn-Ga as a function of temperature with the hope of observing a change in slope around $T_{c}$. (ii) Low temperature (below $30 \mathrm{~K}$ ) measurements of phonon dispersion in $\alpha$-U to observe a possible up-turn (akin to that in Ni-Mn-Ga). (iii) Measurements indicating a possible structural phase transition to a monoclinic martensite around $1.2 \mathrm{~K}$ in $\alpha$-U and possibly a similar transition in $\alpha$-Pu below $60 \mathrm{~K}$.

From the new perspective presented here it is clear that, concerning structural behavior, the underlying physics in such disparate systems as shape memory alloys and certain actinides is quite similar. We have attempted to extract some unifying principles that provide new insight into multistage, modulated structural transformations. Moreover, these connections may enable exchange of concepts and expertise from one set of materials to another and vice versa.

Inclusion of fully three-dimensional strain, shuffle and magnetization gradient term $\mathrm{s}^{50}$ will allow us to study domain walls, especially antiphase boundaries, twin boundaries and microstructure by augmenting the Landau free energy with Ginzburg terms ${ }^{48}$ in multistage transformations. It remains to be explored how the multistage character of the transformation affects domain wall orientation and energetics, specifically the microstructure.

This global view of the many factors implicated for different materials has drawn our attention particularly to the need for further study, in any complex material, for new, refined measurements of heat capacities and magnetic susceptibility.

\section{ACKNOWLEDGMENT}

We thank L. Mañosa, E. Vives, J.C. Lashley, T. Lookman, R.C. Albers, A.R. Bishop, and S.R. Shenoy for fruitful discussions. We are indebted to G.R. Barsch, J.A. Krumhansl, K. Otsuka, and S.M. Shapiro for critical comments. A.S. gratefully acknowledges support from Iberdrola (Spain). This work was supported in part by the U.S. Department of Energy and in part by the CICyT (Spain) Project No. MAT2001-3251 and CIRIT (Catalonia) Project No. 2001SGR00066.
${ }^{1}$ K. Otsuka and C.M. Wayman, Shape Memory Materials (Cambridge University Press, Cambridge, 1998).

${ }^{2}$ It is not yet established whether the modulated regions are true homogeneous thermodynamic phases in the sense of Gibbs. Therefore, modulated structures may be a better descriptor. See Modulated Structures-1979, edited by J. M. Cowley, J. B. Cohen, M. B. Salamon, and B. J. Wuen, AIP Conf. Proc. No. 53 (AIP, New York, 1979).

${ }^{3}$ G.R. Barsch, J.A. Krumhansl, L.E. Tanner, and M. Wuttig, Scr. Metall. 21, 1257 (1987), and references therein.

${ }^{4}$ By precursors we specifically mean structural effects (e.g., modulated phases) above $T_{M}$ while anomalies in phonons, elastic con- stants and other physical variables, above $T_{M}$, are referred to as premonitory effects.

${ }^{5}$ J.A. Krumhansl, Mater. Sci. Forum 327-328, 1 (2000).

${ }^{6}$ S. Kartha, J.A. Krumhansl, J.P. Sethna, and L.K. Wickham, Phys. Rev. B 52, 803 (1995).

${ }^{7}$ S.R. Shenoy, T. Lookman, A. Saxena, and A.R. Bishop, Phys. Rev. B 60, R12 537 (1999); T. Lookman, S.R. Shenoy, K.Ø. Rasmussen, A. Saxena, and A.R. Bishop, ibid. 67, 024114 (2003).

${ }^{8}$ A. Planes and Ll. Mañosa, Solid State Phys. 55, 159 (2001).

${ }^{9}$ For a complete discussion on the physical origin of premartensitic anomalies in elastic moduli and phonon spectra, see M.I. 
Katsnelson, I.I. Naumov, and A.V. Trefilov, Phase Transitions 49, 143 (1994).

${ }^{10}$ J.A. Krumhansl, Solid State Commun. 84, 251 (1992).

${ }^{11} \mathrm{In}_{2} \mathrm{MnGa}$, the IC phase is described as "tweed" contrast. V.V. Kokorin, V.A. Chernenko, J. Pons, C. Segui, and E. Cesari, Solid State Commun. 101, 7 (1997).

${ }^{12}$ V.A. Chernenko, J. Pons, C. Segui, and E. Cesari, Acta Mater. 50, 53 (2002).

${ }^{13}$ A. Zheludev, S.M. Shapiro, P. Wochner, and L.E. Tanner, Phys. Rev. B 54, 15045 (1996).

${ }^{14}$ Ll. Mañosa, A. Planes, J. Zaretsky, T. Lograsso, D.L. Schlagel, and C. Stassis, Phys. Rev. B 64, 024305 (2001).

${ }^{15}$ G.H. Lander, J. Magn. Magn. Mater. 29, 271 (1982).

${ }^{16}$ J.C. Lashley, B.E. Lang, J. Boerio-Goates, V.G. Woodfield, G.M. Schmiedeshoff, E.C. Gay, C.C. McPheeters, D.J. Thoma, W.L. Hults, J.C. Cooley, R.J. Hanrahan, and J.L. Smith, Phys. Rev. B 63, 224510 (2001).

${ }^{17}$ A. Planes, E. Obradó, A. Gonzalez-Comas, and Ll. Mañosa, Phys. Rev. Lett. 79, 3926 (1997).

${ }^{18}$ Ll. Mañosa, A. Gonzalez-Comas, E. Obradó, A. Planes, V.A. Chernenko, V.V. Kokorin, and E. Cesari, Phys. Rev. B 55, 11068 (1997).

${ }^{19}$ E. Obradó, A. Gonzalez-Comas, Ll. Mañosa, and A. Planes, J. Appl. Phys. 83, 7300 (1998).

${ }^{20}$ T.E. Stenger and J. Trivisonno, Phys. Rev. B 57, 2735 (1998).

${ }^{21}$ V.V. Martynov and V.V. Kokorin, J. Phys. III 2, 739 (1992).

${ }^{22}$ S.M. Shapiro, Y. Noda, Y. Fujii, and Y. Yamada, Phys. Rev. B 30, 4314 (1984).

${ }^{23}$ M.B. Salamon, M.E. Meichle, and C.M. Wayman, Phys. Rev. B 31, 7306 (1985).

${ }^{24}$ I. Folkins and M.B. Walker, Phys. Rev. B 40, 255 (1989).

${ }^{25}$ G.R. Barsch, Mater. Sci. Forum 327-328, 367 (2000).

${ }^{26}$ D. Stróz, Scr. Mater. 47, 363 (2002).

${ }^{27}$ D. Shindo, Y. Murakami, and T. Ohba, Mater. Res. Bull. 27, 121 (2002)

${ }^{28}$ X. Ren, N. Miura, K. Taniwaki, K. Otsuka, T. Suzuki, K. Tanaka, Yu.I. Chumlyakov, and M. Asai, Mater. Sci. Eng., A 273-275, 190 (1999).

${ }^{29}$ X. Ren, N. Miura, J. Zhang, K. Otsuka, K. Tanaka, M. Koiwa, T. Suzuki, Yu.I. Chumlyakov, and M. Asai, Mater. Sci. Eng., A 312, 196 (2001).

${ }^{30}$ T. Ohba, S.M. Shapiro, S. Aoki, and K. Otsuka, Jpn. J. Appl. Phys. 33, L1631 (1994); T. Ohba, S. Raymond, S.M. Shapiro, and K. Otsuka, ibid. 37, L64 (1998).

${ }^{31}$ S.M. Shapiro, J.Z. Larese, Y. Noda, S.C. Moss, and L.E. Tanner, Phys. Rev. Lett. 57, 3199 (1986).

${ }^{32}$ S.M. Shapiro, B.X. Yang, G. Shirane, Y. Noda, and L.E. Tanner, Phys. Rev. Lett. 62, 1298 (1989).
${ }^{33}$ S. Muto, S. Takeda, R. Oshima, and F.E. Fujita, Jpn. J. Appl. Phys. 27, L1387 (1988).

${ }^{34}$ S. Muto, R. Oshima, and F.E. Fujita, Acta Metall. Mater. 38, 685 (1990).

${ }^{35}$ S. Muto, R. Oshima, and F.E. Fujita, Metall. Trans. A 19, 2723 (1988).

${ }^{36}$ T.R. Finlayson, M. Mostoller, W. Reichardt, and H.G. Smith, Solid State Commun. 53, 461 (1985).

${ }^{37}$ A. Planes and Ll. Mañosa, Mater. Sci. Forum 327-328, 421 (2000).

${ }^{38}$ H.G. Smith, N. Wakabayashi, W.P. Crummett, R.M. Nicklow, G.H. Lander, and E.S. Fisher, Phys. Rev. Lett. 44, 1612 (1980).

${ }^{39}$ E.S. Fisher and H.J. McSkimin, Phys. Rev. 124, 67 (1961).

${ }^{40}$ For careful measurements of the phonon energy below $30 \mathrm{~K}$ we expect an upturn in $\alpha$-U similar to that for Ni-Mn-Ga.

${ }^{41} \mathrm{In} \mathrm{NiTi}(\mathrm{Fe})$ the phonon freezing is accompanied by a change in symmetry but the upturn has been observed in NiTi. See, for instance, O. Mercier, K.N. Melton, G. Gremaud, and J. Hagi, J. Appl. Phys. 51, 1833 (1980).

${ }^{42}$ S.S. Hecker, Mater. Res. Bull. 26, 672 (2001).

${ }^{43}$ See, e.g., L.E. Tanner, D. Schryvers, and S.M. Shapiro, Mater. Sci. Eng., A 127, 205 (1990).

${ }^{44}$ C.M. Hwang, M. Meichle, M.B. Salamon, and C.M. Wayman, Philos. Mag. A 47, 9 (1983).

${ }^{45}$ T. Castán, E. Vives, and P.-A. Lindgård, Phys. Rev. B 60, 7071 (1999).

${ }^{46}$ M. Sugiyama, Ph.D. thesis, Osaka University, 1985.

${ }^{47}$ A.N. Vasil'ev, A.D. Bozhko, V.V. Khovailo, I.E. Dikhtein, V.G. Shavrov, V.D. Buchelnikov, M. Matsumoto, S. Suzuki, T. Takagi, and J. Tani, Phys. Rev. B 59, 1113 (1999).

${ }^{48}$ G.R. Barsch and J.A. Krumhansl, Phys. Rev. Lett. 53, 1069 (1984); K.Ø. Rasmussen, T. Lookman, A. Saxena, A.R. Bishop, R.C. Albers, and S.R. Shenoy, ibid. 87, 055704 (2001).

${ }^{49}$ Maybe representable by phonons, but ultimately by any complete basis for a lattice, possibly including wavelets. See, e.g., M. Hase, M. Kitajima, S.I. Nakashima, and K. Mizoguchi, Phys. Rev. Lett. 88, 067401 (2002); J.-P. Antoine, Ph. Antoine, and B. Piraux, in Wavelets in Physics, edited by J. C. van den Berg, (Cambridge University Press, Cambridge, 1999).

${ }^{50}$ T. Castán, A. Saxena, and A. Planes (unpublished).

${ }^{51}$ This coupling was first introduced in the context of a reconstructive transition in $\mathrm{Zr}$ by P.-A. Lindgård and O.G. Mouritsen, Phys. Rev. Lett. 57, 2458 (1986).

${ }^{52}$ X. Ren and K. Otsuka, Scr. Mater. 38, 1669 (1998).

${ }^{53}$ S.Y. Savrasov, G. Kotliar, and E. Abrahams, Nature (London) 410, 793 (2001); R.C. Albers, ibid. 410, 759 (2001).

${ }^{54}$ M.B. Walker, Phys. Rev. B 34, 6830 (1986). 